\title{
ON ANALYTIC FUNCTIONS WHICH HAVE ALGEBRAIC VALUES AT A CONVERGENT SEQUENCE OF POINTS $\left.{ }^{(}\right)$
}

\author{
BY \\ D. L. HILLIKER
}

I. Introduction. A function $g(z)$ is algebraic if it satisfies an equation

$$
\sum_{\lambda, \mu=0}^{l, m} c_{\lambda \mu} z^{\lambda}(g(z))^{\mu}=0
$$

with $c_{\lambda \mu}$ constant. If in particular the coefficients are algebraic or, equivalently, rational integral, $g(z)$ is said to be algebraic in the arithmetic sense. Th. Schneider (Schneider [1, Theorems I, III]) in 1951 established criteria under which an analytic function is algebraic in the arithmetic sense. O. S. Içen (Içen [1]) generalized Schneider's results. In this paper we seek other criteria which extend Schneider's theorems and in certain cases, Içen's theorems as well. The results are then generalized to sufficient conditions for $m$ functions to be algebraically dependent. The degree to which these theorems are best possible is discussed.

II. Algebraic functions. The height $H(\theta)$ of an algebraic number $\theta$ is the maximum of the absolute values of the coefficients of the minimal polynomial of $\theta$.

Lemma 1. If $g(z)$ is algebraic in the arithmetic sense, then

$$
g(1 / v) \text { is algebraic of bounded degree for all }
$$

$$
\text { sufficiently large integral } v
$$

and

$$
\limsup _{\nu \rightarrow \infty} \frac{\log H(g(1 / \nu))}{\log \nu}<\infty .
$$

Proof. $g(z)$ satisfies equation (1) with integral coefficients and hence

$$
\sum_{\lambda, \mu=0}^{l, m} c_{\lambda \mu} \nu^{l-\lambda}(g(1 / \nu))^{\mu}=0
$$

Thus (2) holds for all but finitely many values of $\nu$. Using Le Veque's Theorem 4-3 (Le Veque [1, p. 126]) and (4), we obtain

$$
H(g(1 / \nu)) \leqq 6^{m} \max _{\lambda, \mu}\left|c_{\lambda \mu}\right| v^{l-\lambda} .
$$

Presented to the Society, March 26, 1966; received by the editors March 28, 1966.

(1) My greatest thanks and appreciation are given to Professor E. G. Straus at the University of California, Los Angeles, for suggestions made in the preparation of this Ph.D. dissertation. 
Thus there is a positive constant $k$ for which $H(g(1 / v))<\nu^{k}$ for $\nu>\nu_{0}$. Hence (3) holds.

We shall need the following generalization of Lemma 1.

LEMMA 2. If $g(z)$ is algebraic and $g\left(z_{v}\right)$ is algebraic for infinitely many algebraic values of $z_{v}$, then $g(z)$ is algebraic in the arithmetic sense and hence (2) and (3) hold.

Proof. $g(z)$ satisfies equation (1) and hence

$$
\sum_{\lambda, \mu=0}^{l, m} c_{\lambda \mu} z_{v}^{\lambda}\left(g\left(z_{v}\right)\right)^{\mu}=0
$$

We can write

$$
c_{\lambda \mu}=c_{\lambda \mu}^{(0)}+c_{\lambda \mu}^{(1)} \xi_{1}+\cdots+c_{\lambda \mu}^{(k)} \xi_{k}
$$

where the $c_{\lambda \mu}^{(\kappa)}$ 's are algebraic, and the $\xi_{\kappa}$ 's are transcendental and linearly independent over the field of algebraic numbers. Substituting (6) into (5) we obtain

$$
\sum_{\lambda, \mu=0}^{l, m} c_{\lambda \mu}^{(\kappa)} z_{\nu}^{\lambda}\left(g\left(z_{v}\right)\right)^{\mu}=0
$$

Therefore the functions

$$
G^{(\kappa)}(z)=\sum_{\lambda, \mu=0}^{l, m} c_{\lambda \mu}^{(\kappa)} z^{\lambda}(g(z))^{\mu}
$$

have zeros at $z_{v}$. But $G^{(\kappa)}(z)$ is algebraic so that it can have infinitely many zeros only if it is identically zero.

The interesting thing is that for functions which are analytic at $z=0$, the converse of Lemma 1 is also true. In fact (2) or (3) may be weakened. Note that for $g(z)$ analytic at $z=0, g(1 / \nu)$ is defined for sufficiently large $\nu$. We begin by keeping (2) and weakening (3).

THEOREM I. Let $g(z)$ be analytic at $z=0$ and suppose that

$$
\begin{aligned}
& g(1 / \nu) \text { is algebraic of degree at most d } \\
& \text { for sufficiently large integral } \nu
\end{aligned}
$$

$$
\limsup _{\nu \rightarrow \infty} \frac{\log H(g(1 / \nu))}{\nu \log \nu}=y<\frac{1}{20 d^{3}+12 d^{2}-13 d+2} \text {. }
$$

Then $g(z)$ is algebraic in the arithmetic sense. Conversely, if $g(z)$ is algebraic in the arithmetic sense, then there is a d for which (7) holds and (8) holds with $y=0$.

Proof. Let $g(z)$ be analytic in $|z|<r$. We have $1 / \nu<r / 2$ for $\nu>\nu_{0}$ with $\nu_{0}$ integral. Let $t$ be such that $y<t<1 /\left(20 d^{3}+12 d^{2}-13 d+2\right)$. Then by (8) we have, for sufficiently large $\nu$, say $\nu>\nu_{0}$, that

$$
\log H(g(1 / v))<t v \log v
$$


Let

$$
\phi(z)=\sum_{\lambda, \mu=0}^{l, m} c_{\lambda \mu} z^{\lambda}(g(z))^{\mu}
$$

We seek integers $c_{\lambda \mu}$ not all zero and not too large for which

$$
\phi(1 / v)=0 \quad \text { for } \nu=\nu_{0}+1, \ldots, \nu_{0}+n .
$$

Where $l, m$, and $n$ are integers to be specified later. Let $g(1 / v)=\beta_{v}=\beta$ and

$$
\operatorname{deg} g(1 / v)=\delta(v)=\delta \leqq d .
$$

Let the minimal equation for $\beta$ be

$$
\sum_{x=0}^{\delta} b_{x} \beta^{x}=0
$$

so that

$$
\max _{0 \leqq x \leqq \delta}\left|b_{x}\right|=H(g(1 / v)) .
$$

The following lemma is easily established by induction on $\mu$.

LEMMA 3. There are integers $s_{x u}$ for which

$$
\beta^{\mu}=b_{\delta}^{-\mu}\left(s_{0 \mu}+s_{1 \mu} \beta+\cdots+s_{\delta-1 \mu} \beta^{\delta-1}\right)
$$

and

$$
\left|s_{x \mu}\right| \leqq(2 H(\beta))^{\mu} \quad(\chi=0, \ldots, \delta-1 ; \mu=0,1,2, \ldots) .
$$

From (11) and Lemma 3 we obtain a system

$$
\sum_{\lambda, \mu=0}^{l, m} \nu^{l-\lambda} b_{\delta}^{m-\mu} s_{x \mu} c_{\lambda \mu}=0
$$

where $\chi=0, \ldots, \delta-1$ and $\nu=\nu_{0}+1, \ldots, \nu_{0}+n$, of at most $n d$ equations in the $(l+1)(m+1)$ unknowns $c_{\lambda \mu}$.

Let $l=[n / 2(d+1)]$ and $m=4 d(d+1)-1$. Then $(l+1)(m+1) \geqq 2 n d$. For some integer $c(n)$ to be specified later, we seek a solution of $(12)$ for which

$$
\left|c_{\lambda \mu}\right|<n^{\lambda} c(n) \text {. }
$$

Let $c_{\lambda \mu}=0,1, \ldots, n^{\lambda} c(n)-1$. Let $y_{v, \delta}$ denote the linear form on the left of (12). Then using Lemma 3 and (13) we get

$$
\left|y_{v, \delta}\right| \leqq c(n) \exp [l \log n+m t v \log \nu+o(n \log n)] .
$$

The number of $c_{\lambda \mu}$-vectors is

$$
\prod_{\lambda, \mu=0}^{l, m} n^{\lambda} c(n)=n^{l(l+1)(m+1) / 2}(c(n))^{(l+1)(m+1)} .
$$


By (14), the number of $y_{v, \delta}$-vectors is at most

$$
\prod_{\nu=\nu_{0}+1}^{\nu_{0}+n}(c(n) \exp [l \log n+m t \nu \log \nu+o(n \log n)])^{d} .
$$

Thus we see that there is a function $f(n)=o(n \log n)$ such that if $h=(2 d(d+1)-1 / 2) t$ and $c(n)=[\exp (h n \log n+f(n))]$, then the number of $c_{\lambda \mu}$-vectors is greater than the number of $y_{v, \delta}$-vectors. With this choice of $c(n)$ we have two different $c_{\lambda \mu}$-vectors that are mapped into the same $y_{v, \delta}$-vector. Consequently, by the linearity of $y_{v, \delta}$, their difference is mapped into the zero vector. Letting $c_{\lambda \mu}$ denote the components of the difference vector, we see by (13) that (12) has a solution in integers $c_{\lambda \mu}$ not all zero for which

$$
\left|c_{\lambda \mu}\right| \leqq n^{\lambda} \exp [h n \log n+o(n \log n)] .
$$

Since $h>0$, the upper bound in (15) is an increasing function of $n$.

We have $\phi(z)=0$ for

$$
z=1 /\left(\nu_{0}+1\right), \ldots, 1 /\left(\nu_{0}+n\right) .
$$

We shall now show that if $\phi(z)=0$ for

$$
z=1 /\left(\nu_{0}+1\right), \ldots, 1 /\left(\nu_{0}+n_{1}\right),
$$

then $\phi(z)=0$ for $z=1 /\left(\nu_{0}+n_{1}+1\right)$ provided $n_{1}>n$ and $n$ is sufficiently large.

The function

$$
\frac{\phi(z)}{\left(z-1 /\left(\nu_{0}+1\right)\right) \cdots\left(z-1 /\left(\nu_{0}+n_{1}\right)\right)}
$$

is analytic in $|z| \leqq 3 r / 4$. Therefore, by using the maximum principle and the fact that $1 /\left(\nu_{0}+n_{1}+1\right)<3 r / 4$, we get

$$
\begin{array}{r}
\left|\frac{\phi\left(\frac{1}{\nu_{0}+n_{1}+1}\right)}{\left(\frac{1}{\nu_{0}+n_{1}+1}-\frac{1}{\nu_{0}+1}\right) \cdots\left(\frac{1}{\nu_{0}+n_{1}+1}-\frac{1}{\nu_{0}+n_{1}}\right)}\right| \\
\leqq \max _{|z|=3 r / 4}\left|\frac{\phi(z)}{\left(z-\frac{1}{\nu_{0}+1}\right) \cdots\left(z-\frac{1}{\nu_{0}+n_{1}}\right)}\right|
\end{array}
$$

From (10), (15) and the fact that $\lambda \leqq l$, we obtain

$$
|\phi(z)| \leqq \exp \left[\left(\frac{1}{2(d+1)}+h\right) n_{1} \log n_{1}+o\left(n_{1} \log n_{1}\right)\right]
$$

for $|z|=3 r / 4$. Also for $|z|=3 r / 4$ we have

$$
\left|z-1 /\left(\nu_{0}+1\right)\right| \cdots\left|z-1 /\left(\nu_{0}+n_{1}\right)\right| \geqq(r / 4)^{n_{1}} .
$$


Using $n_{1}^{n} 1 e^{-n_{1}}<n_{1} ! \leqq n_{1}^{n_{1}}$ we obtain

$$
\left|\frac{1}{\nu_{0}+n_{1}+1}-\frac{1}{\nu_{0}+1}\right| \cdots\left|\frac{1}{\nu_{0}+n_{1}+1}-\frac{1}{\nu_{0}+n_{1}}\right| \leqq \gamma^{n_{1} n_{n_{1}}^{-n_{1}}}
$$

for some positive constant $\gamma$. Putting (17), (18), and (19) into (16) yields

$$
\left|\phi\left(\frac{1}{\nu_{0}+n_{1}+1}\right)\right| \leqq \exp \left[\left(\frac{1}{2(d+1)}+h-1\right) n_{1} \log n_{1}+o\left(n_{1} \log n_{1}\right)\right] .
$$

By using (10), (15), and (9), we get for the conjugates

$$
\phi^{(x)}\left(\frac{1}{\nu_{0}+n_{1}+1}\right) \text { of } \phi\left(\frac{1}{\nu_{0}+n_{1}+1}\right)
$$

the upper bound

$$
\left|\phi^{(\kappa)}\left(\frac{1}{\nu_{0}+n_{1}+1}\right)\right| \leqq \exp \left[\{h+(4 d(d+1)-1) t\} n_{1} \log n_{1}+o\left(n_{1} \log n_{1}\right)\right] .
$$

Let $K$ be the denominator of $\phi\left(1 /\left(\nu_{0}+n_{1}+1\right)\right)$. Then by (10) and (9),

$$
K \leqq \exp \left[\left\{\frac{1}{2(d+1)}+(4 d(d+1)-1) t\right\} n_{1} \log n_{1}+o\left(n_{1} \log n_{1}\right)\right]
$$

By using (20), (21), and (22), we get for the norm of the algebraic integer

$$
K \phi\left(1 /\left(\nu_{0}+n_{1}+1\right)\right)
$$

$$
\begin{aligned}
& \left|N\left(K \phi\left(\frac{1}{\nu_{0}+n_{1}+1}\right)\right)\right| \\
& \leqq \exp \left[\left\{-\frac{1}{2}+\left(10 d^{3}+6 d^{2}-\frac{13}{2} d+1\right) t\right\} n_{1} \log n_{1}+o\left(n_{1} \log n_{1}\right)\right] .
\end{aligned}
$$

Thus by (8), the norm has absolute values less than 1 for all $n_{1} \geqq n$ if $n$ is sufficiently large.

With this choice of $n$, we have

$$
\phi\left(1 /\left(\nu_{0}+n_{1}+1\right)\right)=0 \quad \text { for } n_{1} \geqq n
$$

and consequently

$$
\phi(z)=0 \quad \text { for } z=1 /\left(v_{0}+1\right), 1 /\left(\nu_{0}+2\right), \ldots
$$

This implies, since $\phi(z)$ is analytic at $z=0$, that $\phi(z) \equiv 0$. Consequently $g(z)$ is algebraic in the arithmetic sense.

We remark that the upper bound in (8) which is asymptotic to $1 / 20 d^{3}$, can be improved by a better choice of $l$ and $m$ to one which is asymptotic to $1 / 18.6 d^{3}$.

Theorem I generalizes Schneider's Theorem I. It also generalizes Içen's Theorem I when specialized to the case $z_{v}=1 / \nu$ and $g(z)$ a function of a complex variable.

A series of the form

$$
f(z)=\sum_{x=0}^{\infty} a_{x}\left(z-z_{1}\right) \cdots\left(z-z_{x}\right)
$$

where $\left\{z_{v}\right\}$ is a sequence of complex numbers, is called a generalized Taylor series. 
LEMMA 4. If $f(z)$ is analytic for $|z|<r$ and $\left|z_{v}\right| \leqq \delta r$ for $\nu=1,2,3, \ldots$; then the series (24) converges for $|z|<(1-2 \delta) r$ and $\left|a_{x}\right| \leqq c((1-\delta) r)^{-x}$, for $\chi>\chi_{0}$, where $c$ is some positive constant.

LEMMA 5. If

$$
\limsup _{x \rightarrow \infty}\left(\left|a_{x}\right|\right)^{1 / x}=\frac{1}{R} \text { and } \limsup _{x \rightarrow \infty}\left|z_{x}\right|=\delta R,
$$

then the series (24) converges for $|z|<(1-\delta) R$ to an analytic function.

Lemma 6. Let $\alpha$ and $\beta$ be algebraic numbers of degree $s_{1}$ and $s_{2}$. Then

$$
H(\alpha+\beta) \leqq c(H(\alpha))^{s_{2}}(H(\beta))^{s_{1}}
$$

where $c>0$ depends only on $s_{1}$ and $s_{2}$. If $\alpha \neq 0$, then

$$
|\alpha| \geqq 1 / 2 H(\alpha) \text {. }
$$

We omit the proofs of Lemmas 4 and 5, but we shall indicate why (25) holds. Let $\theta$ be an algebraic number with degree $s$, denominator $a$ and conjugates $\theta=\theta^{(1)}, \theta^{(2)}, \ldots, \theta^{(s)}$. Define

$$
H^{*}(\theta)=|a| \prod_{i=1}^{s} \max \left(1,\left|\theta^{(i)}\right|\right)
$$

Then by Lang's Lemma 1 (Lang [1, p. 47]),

$$
2^{-s} H^{*}(\theta) \leqq H(\theta) \leqq 2^{s} H^{*}(\theta)
$$

Also we have

$$
\begin{aligned}
H^{*}(\alpha+\beta) & \leqq|a b| \prod_{i, j} \max \left(1,\left|\alpha^{(i)}+\beta^{(j)}\right|\right) \\
& \leqq|a b| \prod_{i, j}\left(2 \max \left(1,\left|\alpha^{(i)}\right|\right) \max \left(1,\left|\beta^{(j)}\right|\right)\right)=2^{s_{1} s_{2}} H^{*}(\alpha) H^{*}(\beta)
\end{aligned}
$$

and this combined with (26) yields (25).

We shall investigate the degree to which Theorem I is best possible.

THEOREM II. Let $y$ be defined by (8). Then for $y=1$ there are uncountably many (transcendental) functions $g(z)$ for which

$$
g(z) \text { is analytic at } z=0 .
$$

For a sufficiently large integral $\nu$, $g(1 / v)$ is algebraic of degree at most $d$.

The set of functions satisfying (27), (28), and $y<1 / 2 d$ is countable. 
Proof. We first prove the second assertion. Let $g(z)$ be analytic in $|z|<r$. Then we have $1 / \nu<r / 4$ for $\nu>\nu_{0}$ and

$$
g(z)=\sum_{x=0}^{\infty} a_{x}\left(z-\frac{1}{v_{0}+1}\right)\left(z-\frac{1}{\nu_{0}+2}\right) \cdots\left(z-\frac{1}{v_{0}+\chi}\right)
$$

for $|z|<r / 2$.

Let

$$
R_{v}=\sum_{x=0}^{\nu-\nu_{0}-2} a_{x} \frac{\left(\nu_{0}+1-\nu\right) \cdots\left(\nu_{0}+\chi-\nu\right)}{\nu^{x}\left(\nu_{0}+1\right) \cdots\left(\nu_{0}+\chi\right)}
$$

Then for $\nu>\nu_{1}$ with $\nu_{1}$ sufficiently large and for some constant $c_{1}>0$,

$$
\left|R_{v}-g(1 / \nu)\right| \leqq\left(c_{1} \nu\right)^{-\left(\nu-v_{0}-1\right)}
$$

The coefficients $a_{0}, \ldots, a_{x}$ are determined once

$$
g\left(1 /\left(\nu_{0}+1\right)\right), \ldots, g\left(1 /\left(\nu_{0}+\chi+1\right)\right)
$$

have been chosen. Suppose that there are two distinct choices $\alpha$ and $\beta$ of $g(1 / v)$ for $\nu>\nu_{1}$. Then by (30),

$$
1 / 2 H(\alpha-\beta)<|\alpha-\beta| \leqq 2\left(c_{1} \nu\right)^{-\left(\nu-v_{0}-1\right)} .
$$

Let $\varepsilon>0$. Then from (31), Lemma 6 and (8) we infer that for $\nu$ sufficiently large,

$$
c_{2} \nu^{2 d(y+\varepsilon) \nu}>\left(c_{1} \nu\right)^{\nu-v_{0}-1}
$$

where $c_{2}>0$ depends only on $d$. But for $\varepsilon$ sufficiently small, (32) contradicts $y<1 / 2 d$. Thus, there is at most one choice for $g(1 / \nu)$ for $\nu>\nu_{1}$. Consequently the denumerable number of choices for $g(1 / \nu), \nu \leqq \nu_{1}$ determines $g(z)$ and there are only countably many such functions $g(z)$.

Now let us prove the first part of the theorem. We seek functions $g(z)$ given by (29) satisfying (28) and such that

$$
\limsup _{v \rightarrow \infty} \frac{\log H(g(1 / v))}{\nu \log v}=1 .
$$

Let $a_{x}=\varepsilon_{\chi} / 2^{x}(\chi+1)$ and $\nu_{0}=0$ where $\varepsilon_{x}$ can be chosen in two different ways as, say, 1 or -1 . Then (29) converges for $|z|<2$. Consequently $g(1 / v)$ is bounded and

$$
g(1 / v)=\sum_{\chi=0}^{\nu-1} \frac{(-1)^{x} \varepsilon_{x}\left(\begin{array}{l}
\nu-1 \\
\chi+1
\end{array}\right)}{(2 v)^{x}}<M
$$

for some constant $M$. Moreover, $g(1 / v)$ is rational with denominator $(2 v)^{v-1}$ so that (33) holds. Thus (29) yields uncountably many such functions $g(z)$ all of which are transcendental by Lemma 2 . This completes the proof of Theorem II. 
Thus we see that if (8) could be replaced by the condition $y<1$, then the resulting theorem would be best possible.

Let us now consider functions $g(z)$ which satisfy (27) and (28). We know by Lemma 2 that if $g(z)$ is algebraic, then $y=0$. Conversely, by Theorem I we know that if $y=0$, then $g(z)$ is algebraic. Thus Theorem I tells us that there are no such functions $g(z)$ with $0<y<1 /\left(20 d^{3}+12 d^{2}-13 d+2\right)$. Following E. G. Straus, we define the arithmetic limit of $y$ to be that number $y_{0}$ such that for each $y \geqq y_{0}$ there are uncountably many such functions $g(z)$ and for all $y<y_{0}$ there are at most countably many such functions $g(z)$. Thus, by Theorem II, we have $1 / 2 d \leqq y_{0} \leqq 1$ if $y_{0}$ exists. See Figure 1.

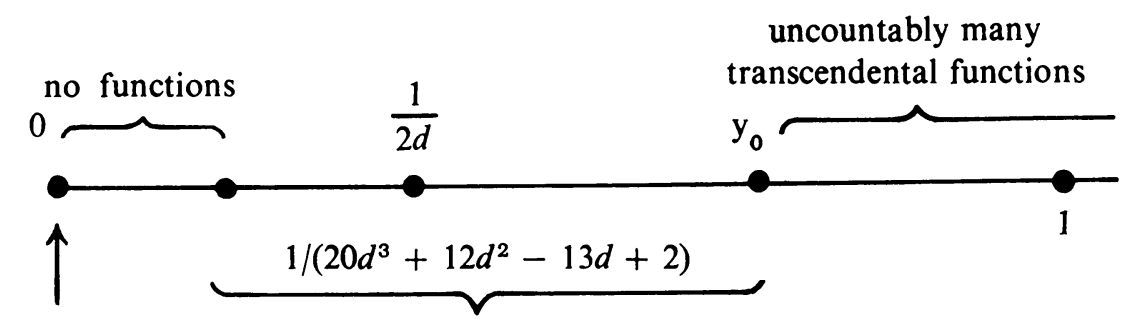

all

at most countably many

algebraic transcendental functions

functions

FIGURE 1. The spectrum on the $y$-axis of functions $g(z)$ satisfying (27) and (28).

We now weaken condition (2). By Schneider's Lemma 29 (Schneider [3, p. 140]) and an argument similar to that in the proof of Theorem I, we obtain the following.

THEOREM III. Let $g(z)$ be analytic at $z=0$. Suppose that $g(1 / v)$ is algebraic, deg $g\left(1 /\left(\nu_{0}+\nu\right)\right) \leqq d_{v}$ for integral $\nu>\nu_{0}$ and $d_{v}$ nondecreasing

and

$$
\lim _{\nu \rightarrow \infty} \frac{d_{v+1}^{3} \log 3 H(g(1 / \nu))}{\nu \log \nu}=0 .
$$

Then $g(z)$ is algebraic in the arithmetic sense. Conversely, if $g(z)$ is algebraic in the arithmetic sense, then the degree is bounded and there is a $d_{v}$ for which (34) and (35) hold.

Theorem III generalizes Schneider's Theorems I and III $\left({ }^{2}\right)$. Also if we let $z_{v}=1 / v$ in Içen's Theorem I and $z_{v}=1 / \nu, \gamma=(1 / 3) \mathbf{J}$ in Içen's Theorem II, then the resulting specializations of Içen's Theorems I and II are covered in the case that $g(z)$ is a function of a complex variable.

${ }^{(2)}$ Schneider's theorem reads $d_{v}=o\left(\nu^{1 / 2}\right)$ but according to a remark in Içen's paper (Içen $[1, \mathrm{p} .45]$ ) Schneider has announced that this should be $d_{v}=o\left(\nu^{1 / 3}\right)$. 
III. Algebraically dependent functions. We now consider $m$ functions $f_{1}(z), \ldots$, $f_{m}(z)$ which are analytic at one point which we might as well assume to be $z=0$. We assume that the values of these functions at a sequence $\left\{z_{v}\right\}$ which converge to, say, 0 are algebraic. Roughly speaking, if $H\left(f_{\mu}\left(z_{v}\right)\right)$ and $\operatorname{deg} f_{\mu}\left(z_{v}\right)$, for $\mu=1, \ldots, m$ and $\nu=\nu_{0}+1, \nu_{0}+2, \ldots$, are not too large and if the sequence $\left\{z_{v}\right\}$ converges sufficiently rapidly, then $f_{1}(z), \ldots, f_{m}(z)$ are algebraically dependent.

It would be possible to generalize our results to cases in which the sequence $\left\{z_{v}\right\}$ has more than one cluster point in the domain of analyticity of the functions in question.

Let $Q$ be the field of rational numbers. For some index $v_{0}$, let

$$
\operatorname{deg} Q\left(f_{1}\left(z_{v_{0}+v}\right), \ldots, f_{m}\left(z_{v_{0}+v}\right)\right) \leqq d_{v} .
$$

Let

$$
\prod_{\mu=1}^{m} \operatorname{deg} f_{\mu}\left(z_{v}\right) \leqq h_{v}, \quad D_{v}=\sum_{x=v_{0}+1}^{\nu_{0}+v} h_{x}
$$

and

$$
P_{v}=\left|z_{v_{0}+v+1}-z_{v_{0}+1}\right| \cdots\left|z_{v_{0}+v+1}-z_{v_{0}+v}\right| \text {. }
$$

We define a measure $\rho$ and a finer measure $\sigma$ of the asymptotic behavior of $P_{v}$ by

$$
\rho=\liminf _{v \rightarrow \infty}\left(\log \left|\log P_{v}\right| / \log v\right)
$$

and

$$
\sigma=\lim _{\nu \rightarrow \infty} \inf \left(\left|\log P_{v}\right| / \nu^{\rho} \log \nu\right) .
$$

Thus $\rho$ and $\sigma$ are measures of the rate of convergence of $\left\{z_{v}\right\}$. For $\sigma$ we have $0 \leqq \sigma \leqq \infty$, but for $\rho$ we have

LEMMA 7. If $\left\{z_{v}\right\}$ is a convergent sequence of distinct points, then

$$
\lim _{\nu \rightarrow \infty}\left(\left(\log P_{v}\right) / v\right)=-\infty
$$

and consequently $1 \leqq \rho \leqq \infty$.

Proof. Without loss of generality assume that $\left\{z_{v}\right\}$ converges to 0 . Let $\varepsilon>0$. Then there is a $\nu_{1}$ such that

$$
\left|z_{v_{0}+v+1}-z_{v_{0}+\chi}\right|<\varepsilon \quad \text { if } \nu, \chi>v_{1} .
$$

Consequently, $\log P_{\nu}<-\gamma \nu$ for all large $\nu$ and for every $\gamma>0$.

ExAmples. Assume $\nu_{0}=0$. Let $z_{v}=1 / v$. Then $P_{v}=(1+v)^{-v}$. Hence $\rho=1$ and $\sigma=1$. Let $z_{\nu}=\nu^{-1 / 2}$. Then

$$
\left(4 e^{1 / 2}\right)^{-v} v^{-v / 2}<P_{v}<v^{-v / 2}
$$


Hence $\rho=1$ and $\sigma=1 / 2$. More generally, if $\left\{z_{v}\right\}$ is a convergent sequence of distinct algebraic numbers of bounded degree with $H\left(z_{v}\right)=O\left(\nu^{a}\right)$ for some constant $a$, then $\rho=1$. For the sequence $1 / 2^{v}$ we have

$$
\frac{\log 2}{2}\left(v^{2}+v\right)<\left|\log P_{v}\right|<\frac{\log 2}{2}\left(v^{2}+3 v+2\right) .
$$

Hence $\rho=2$ and $\sigma=0$.

Let

$$
\alpha=\limsup _{v \rightarrow \infty} \frac{\log \left(D_{v}^{1 / m} d_{v+1}\right)}{\log \nu}
$$

and

$$
\gamma_{\mu}=\limsup _{\nu \rightarrow \infty} \frac{\log \log 3 H\left(f_{\mu}\left(z_{v}\right)\right)}{\log \nu} .
$$

THEOREM IV. Let $f_{1}(z), \ldots, f_{m}(z)$ be $m$ functions analytic at $z=0$. Let $\left\{z_{v}\right\}$ be a sequence of distinct points converging to zero. Suppose that $f_{\mu}\left(z_{v}\right)$ is algebraic for $\mu=1, \ldots, m$ and $\nu=1,2,3, \ldots ; h_{\nu}$ is a nondecreasing function and that

$$
\alpha+\frac{1}{m} \sum_{\mu=1}^{m} \gamma_{\mu}<\rho .
$$

Then $f_{1}(z), \ldots, f_{m}(z)$ are algebraically dependent.

Proof. We shall only outline the proof since it is similar to that of Theorem I. Let $f_{\mu}\left(z_{v}\right)$ for $\mu=1, \ldots, m$ be analytic in $|z|<r$. We have $\left|z_{v}\right|<r / 2$ if $\nu>\nu_{0}$. Let

$$
\phi(z)=\sum_{\tau_{\mu}=0}^{t_{\mu}} c_{\tau_{1} \cdots \tau_{m}}\left(f_{1}(z)\right)^{\tau_{1}} \cdots\left(f_{m}(z)\right)^{\tau_{m}} .
$$

We seek integers $c_{\tau_{1} \cdots \tau_{m}}$ not all zero and not too large so that $\phi(z)=0$ for $z=z_{v+1}, \ldots, z_{v_{0}+n}$. That is

$$
\sum_{\tau_{\mu}=0}^{t_{\mu}} c_{\tau_{1} \cdots \tau_{m}}\left(f_{1}\left(z_{v}\right)\right)^{\tau_{1}} \cdots\left(f_{m}\left(z_{v}\right)\right)^{\tau_{m}}=0
$$

for $\nu=\nu_{0}+1, \ldots, \nu_{0}+n$.

Let $\beta_{\mu}=\beta_{\mu}(\nu)=f_{\mu}\left(z_{v}\right), \delta(\mu)=\delta(\mu, \nu)=\operatorname{deg} \beta_{\mu}$ and $b_{\delta(\mu)}=b_{\delta(\mu)}(\nu)$ be the denominator of $\beta_{\mu}$ for $\mu=1, \ldots, m$. Then by applying Lemma 3 to each of the algebraic numbers $\beta_{1}^{\tau_{1}}, \ldots, \beta_{m}^{\tau_{m}}$ we obtain

$$
\beta_{1}^{\tau_{1}} \cdots \beta_{m}^{\tau_{m}}=b_{\delta(1)}^{-\tau_{1}} \cdots b_{\delta(m)}^{-\tau_{m}} \sum_{\sigma_{\mu}=0}^{\delta(\mu)-1} a\left(\tau_{1}, \ldots, \tau_{m}, \sigma_{1}, \ldots, \sigma_{m}\right) \beta_{1}^{\sigma_{1}} \ldots \beta_{m}^{\sigma_{m}}
$$

where the coefficients are integers which satisfy

$$
\left|a\left(\tau_{1}, \ldots, \tau_{m}, \sigma_{1}, \ldots, \sigma_{m}\right)\right| \leqq \prod_{\mu=1}^{m}\left(2 H\left(\beta_{\mu}\right)\right)^{\tau_{\mu}} .
$$


If we substitute (38) into (37) and set equal to zero the resulting coefficients of $\beta_{1}^{\tau_{1}} \cdots \beta_{m}^{\tau_{m}}$ we obtain

$$
\sum_{\tau_{\mu}=0}^{\tau_{\mu}} b_{\delta(1)}^{t_{1}-\tau_{1}} \ldots b_{\delta(m)}^{t_{m}-\tau_{m}} a\left(\tau_{1}, \ldots, \tau_{m}, \sigma_{1}, \ldots, \sigma_{m}\right) c_{\tau_{1} \cdots \tau_{m}}=0
$$

for $\nu=\nu_{0}=1, \ldots, \nu_{0}+n ; \sigma_{\mu}=0, \ldots, \delta(\mu)-1 ; \mu=1, \ldots, m$. Thus we have a system (40) of at most $D_{n}$ equations with integral coefficients in the $\prod_{\mu=1}^{m}\left(t_{\mu}+1\right)$ unknowns $c_{\tau_{1} \cdots \tau_{m}}$.

Let $\varepsilon>0$ and $\gamma_{\mu}^{\prime}=\gamma_{\mu}+\varepsilon$, then for $\nu_{0}$ sufficiently large we have

$$
\log 3 H\left(f_{\mu}\left(z_{v}\right)\right)<\left(\nu_{0}+n\right)^{\gamma_{\mu}^{\prime}}
$$

for $\nu=\nu_{0}+1, \ldots, v_{0}+n$. Let

$$
t_{\mu}=\left[\left(2 D_{n}\right)^{1 / m}\left(\nu_{0}+n\right)^{\Upsilon}\right],
$$

where $\Upsilon=m^{-1} \sum_{k=1}^{m} \gamma_{k}^{\prime}-\gamma_{\mu}^{\prime}$. We can assume without loss of generality that

$$
\gamma_{\mu} \leqq \frac{1}{m} \sum_{k=1}^{m} \gamma_{k}+1 / m \quad(\mu=1, \ldots, m) .
$$

For suppose that $\gamma_{1} \leqq \gamma_{2} \leqq \cdots \leqq \gamma_{m}, m \neq 1$ and that (43) does not hold. Then $\gamma_{1}+\cdots+\gamma_{m-1}<(m-1)(\rho-\alpha)$ and (36) holds with $m$ replaced by $m-1$. Now, $D_{n} /\left(\nu_{0}+n\right)$ is a nondecreasing function of $n$ since $h_{v}$ is a nondecreasing function. Consequently, by (43), $t_{\mu}$ is a nondecreasing function of $n$ for which

$$
\prod_{\mu=1}^{m}\left(t_{\mu}+1\right) \geqq 2 D_{n} .
$$

Let $\alpha^{\prime}=\alpha+\varepsilon$ and assume that $\varepsilon$ is small enough so that

$$
\frac{1}{m} \sum_{\mu=1}^{m} \gamma_{\mu}^{\prime}<\rho-\alpha^{\prime} .
$$

It follows from (41), (42), and (45) that

$$
t_{\mu} \log 3 H\left(f_{\mu}\left(z_{v}\right)\right) \leqq c_{1} D_{n}^{1 / m_{n} \rho-\alpha^{\prime}}
$$

for $\nu=\nu_{0}+1, \ldots, \nu_{0}+n$ and

$$
t_{\mu} \leqq c_{1} D_{n}^{1 / m_{n} \rho-\alpha^{\prime}}
$$

where $c_{1}$ and all of the $c_{i}$ 's to follow are positive constants. The coefficients of $c_{\tau_{1} \cdots \tau_{m}}$ in equation (40) have, by (39) and (46), absolute value at most

$$
\prod_{\mu=1}^{m}\left\{2 H\left(f_{\mu}\left(z_{v}\right)\right)\right\}^{t_{\mu}} \leqq \exp \left[c_{2} D_{n}^{1 / m_{n} \rho-\alpha^{\prime}}\right] .
$$


Hence by (44), (48), (47) and Schneider's Lemma 29 (Schneider [3, p. 140]) we see that there are integers $c_{\tau_{1} \cdots \tau_{m}}$ not all zero for which

$$
\left|c_{\tau_{1} \cdots \tau_{m}}\right| \leqq \exp \left[c_{3} D_{n}^{1 / m_{n} \rho-\alpha^{\prime}}\right]
$$

This upper bound is an increasing function of $n$ since by (45), $\alpha^{\prime}<\rho$.

We now assume that $\phi(z)=0$ for $z=z_{v_{0}+1}, \ldots, z_{v_{0}+n_{1}}$ for $n_{1} \geqq n$. By considering the function

$$
\frac{\phi(z)}{\left(z-z_{v_{0}+1}\right) \cdots\left(z-z_{v_{0}+n_{1}}\right)}
$$

which is analytic in $|z| \leqq 3 r / 4$, and the fact that

$$
\left|z_{v_{0}+n_{1}+1}-z_{v_{0}+1}\right| \cdots\left|z_{v_{0}+n_{1}+1}-z_{v_{0}+n_{1}}\right| \leqq \exp \left[-n_{1}^{\rho-\varepsilon / 2} \log n_{1}\right]
$$

we conclude, by the maximum principle, that

$$
\left|\phi\left(z_{v_{0}+n_{1}+1}\right)\right| \leqq \exp \left[c_{4} D_{n_{1}}^{1 / m} n_{1}^{\rho-\alpha^{\prime}}+c_{5} n_{1}-n_{1}^{\rho-\varepsilon / 2} \log n_{1}\right] .
$$

For the denominator $K$ of $\phi\left(z_{v_{0}+n_{1}+1}\right)$ we have

$$
K \leqq \exp \left[c_{6} D_{n_{1}}^{1 / m} n_{1}^{\rho-\alpha^{\prime}}\right]
$$

For the conjugates we have

$$
\left|\phi^{(\delta)}\left(z_{v_{0}+n_{1}+1}\right)\right| \leqq \exp \left[c_{7} D_{n_{1}}^{1 / m} n_{1}^{\rho-\alpha^{\prime}}\right] .
$$

By (51), (52), and (53) we see that the norm satisfies

$$
\left|N\left(K \phi\left(z_{v_{0}+n_{1}+1}\right)\right)\right| \leqq \exp \left[c_{8} d_{n_{1}+1} D_{n_{1}}^{1 / m} n_{1}^{\rho-\alpha^{\prime}}+c_{5} n_{1}-n_{1}^{\rho-\varepsilon / 2} \log n_{1}\right] .
$$

Thus for $\varepsilon$ sufficiently small the last term in the exponent dominates the others so that $\left|N\left(K \phi\left(z_{v_{0}+n_{1}+1}\right)\right)\right|<1$ in the case $\rho \neq 1$ provided $n>n_{0}$ and $n_{0}$ is sufficiently large. Hence $\phi\left(z_{v_{0}+n_{1}+1}\right)=0$ for $n_{1} \geqq n$. Consequently $\phi(z)=0$ for $z=z_{v_{0}+1}$, $z_{v_{0}+2}, \ldots$ so that $\phi(z) \equiv 0$. Thus $f_{1}(z), \ldots, f_{m}(z)$ are algebraically dependent if $\rho \neq 1$. Now assume that $\rho=1$. By Lemma 7 we have

$$
P_{n_{1}} \leqq \exp \left[-\gamma n_{1}\right]
$$

for $n$ sufficiently large. Here $\gamma$ is independent of $n_{1}$ and can be made arbitrarily large. By using (54) in place of (50) we conclude once again that

$$
\left|N\left(K \phi\left(z_{v_{0}+n_{1}+1}\right)\right)\right|<1 \quad \text { for } n>n_{0} .
$$

This completes the proof.

Theorem IV generalizes Schneider's Theorem I. Indeed, let $m=2, f_{1}(z)=g(z)$, $f_{2}(z)=z, z_{\nu}=1 / \nu$ and $\operatorname{deg} g(1 / \nu) \leqq d$. Then $\alpha=1 / 2, \rho=1, H(1 / \nu)=\nu, \gamma_{2}=0$ and the hypothesis becomes $\gamma_{1}<1$ which is exactly Schneider's condition. Consequently $g(z)$ is algebraic (in the arithmetic sense), provided it is analytic at $z=0$. In fact from Theorem IV we get the following generalization of Schneider's Theorem I: 
Let $g(z)$ be analytic at $z=0$. Let $\left\{z_{v}\right\}$ be a sequence of distinct algebraic points converging to zero. Suppose that $g\left(z_{v}\right)$ is algebraic for $\nu=1,2,3, \ldots$ and that

$$
\begin{aligned}
& \limsup _{v \rightarrow \infty} \frac{\log \left(\operatorname{deg} z_{v} \operatorname{deg} g\left(z_{v}\right)\right)}{\log \nu}=\beta, \\
& \limsup _{v \rightarrow \infty} \frac{\log \log 3 H\left(z_{v}\right)}{\log v}=0, \\
& \limsup _{v \rightarrow \infty} \frac{\log \log 3 H\left(g\left(z_{v}\right)\right)}{\log v}<2 \rho-3 \beta-1 .
\end{aligned}
$$

Then $g(z)$ is algebraic in the arithmetic sense.

In Theorem IV the sequence $\left\{z_{v}\right\}$ is completely general except that it converges and the points are distinct. In Içen's paper separate conditions were imposed on the height and degree of $f_{\mu}\left(z_{v}\right)$ and more stringent conditions were imposed on the sequence $\left\{z_{v}\right\}$. In this paper we seek single restrictions on the combined behavior of $\operatorname{deg} f_{\mu}\left(z_{v}\right), H\left(f_{u}\left(z_{v}\right)\right)$ and the rate of convergence of the sequence $\left\{z_{v}\right\}$.

Let $m=2, f_{1}(z)=g(z), f_{2}(z)=z$ and $z_{v}=1 / \nu$. Suppose that

$$
\limsup _{\nu \rightarrow \infty} \frac{\log (\operatorname{deg} g(1 / \nu))}{\log \nu}=\beta .
$$

Then condition (36) is replaced by $3 \beta+\gamma_{1}<1$.

THEOREM V. Let $\beta$ and $\gamma_{1}$ be as above. Then for each $\gamma_{1} \geqq 1$ there are uncountably many (transcendental) functions $g(z)$ such that

$$
g(z) \text { is analytic at } z=0 \text {, }
$$

The set of functions $g(z)$ with $g(1 / v)$ algebraic which satisfy $\beta+\gamma_{1}<1$ and (55) is countable. In particular, in the case $\beta=0$ the arithmetic limit of $\gamma_{1}$ is 1 and hence, in this case Schneider's upper bound for $\gamma_{1}$, namely 1 , is the best possible.

The proof is analogous to that of Theorem II only here the coefficient $a_{x}$ in (33) is given by, for example,

$$
a_{x}=\varepsilon_{x} / 2^{\left[x^{\left.\gamma_{1}\right]}\right.}(\chi+1)
$$

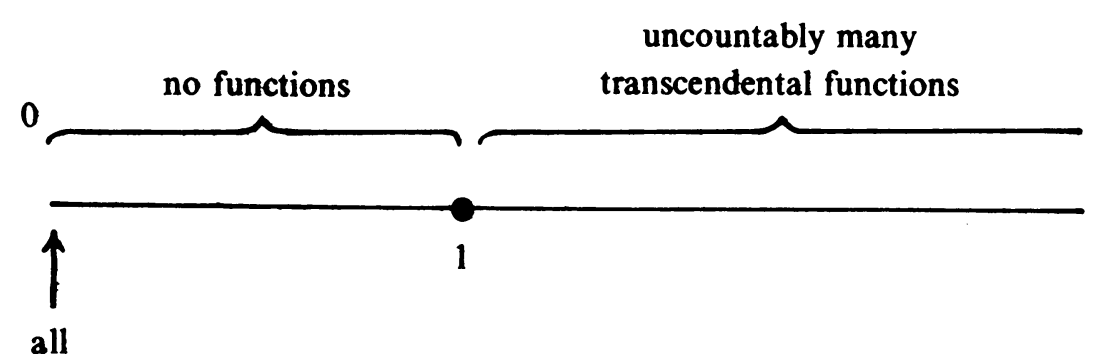

algebraic

functions

FIGURE 2. The spectrum on the $\gamma_{2}$-axis of functions satisfying (55) and (56). 
Thus the spectrum of functions $g(z)$ satisfying (55) and (56) is quite simple. Such a function is algebraic if and only if $\gamma_{1}=0$ and there are no such functions with $0<\gamma_{1}<1$. See Figure 2. Figure 1 is a refinement of Figure 2 indicating what happens at the point $\gamma_{1}=1$.

The following refinement of Theorem IV covers Schneider's Theorem III and can be established by a similar argument.

THEOREM VI. Suppose that $\sigma \neq 0$. Then the conclusion of Theorem IV holds under the same hypotheses but with (36) replaced by

$$
\begin{gathered}
\alpha+\frac{1}{m} \sum_{\mu=1}^{m} \gamma_{\mu}=\rho, \\
\log H\left(f_{\mu}\left(z_{v}\right)\right)=O\left(\nu^{\gamma_{\mu}} \log v\right)
\end{gathered}
$$

for $\mu=1, \ldots, m ;$ and

$$
D_{v}^{1 / m} d_{v+1}=o\left(v^{\alpha}\right) .
$$

To cover Theorem I and Theorem III we need more complicated refinements of condition (36) than that of Theorem VI, namely (a) and (b) below. Let

$$
d_{v}^{*}=\max _{1 \leqq x \leqq \nu}\left(d_{v_{0}+x}\right)
$$

For constants $\alpha_{1}, \ldots, \alpha_{m}$ define

$$
\begin{gathered}
\omega_{\mu}=\limsup _{\nu \rightarrow \infty} \frac{\log \left\{D_{v^{\mu}}^{\alpha_{\mu} / m}\left(d_{v+1}^{*}\right)^{\alpha_{\mu}} \log 3 H\left(f_{\mu}\left(z_{v}\right)\right)\right\}}{\log \nu}, \\
\Omega_{\mu}=\limsup _{\nu \rightarrow \infty} \frac{D_{v^{\mu}}^{\alpha_{\mu} / m}\left(d_{v+1}^{*}\right)^{\alpha_{\mu}} \log 3 H\left(f_{\mu}\left(z_{v}\right)\right)}{\nu^{\omega_{\mu}} \log \nu}, \\
\Delta_{\mu}=\limsup _{\nu \rightarrow \infty} \frac{D_{v^{\mu}}^{\alpha^{\prime} / m}\left(d_{\nu+1}^{*}\right)^{\alpha_{\mu}}}{\nu^{\omega_{\mu}} \log \nu}
\end{gathered}
$$

and

$$
\Gamma_{\mu}(\nu)=D_{v^{\mu}}^{\alpha^{\prime} / m}\left(\nu_{0}+\nu\right)^{\rho-\omega_{\mu}}\left(d_{v+1}^{*}\right)^{\alpha_{\mu}-1} .
$$

THeOREM VII. Let $f_{1}(z), \ldots, f_{m}(z)$ be $m$ functions analytic at $z=0$. Let $\left\{z_{v}\right\}$ be a sequence of distinct points converging to zero. Suppose that $f_{\mu}\left(z_{v}\right)$ is algebraic for $\mu=1, \ldots, m$ and $\nu=1,2,3, \ldots$ Then each of the following two conditions (a) and (b) implies that $f_{1}(z), \ldots, f_{m}(z)$ are algebraically dependent.

(a) There are constants $\alpha_{1}, \ldots, \alpha_{m}$ for which $\sum_{\mu=1}^{m} \alpha_{\mu} \geqq m$,

$$
\begin{gathered}
\max _{1 \leqq x \leqq \nu} \Gamma_{\mu}(\chi) \leqq a \Gamma(v) \quad \text { for } \mu=1, \ldots, m \text { and for some constant } a, \\
\frac{1}{m} \sum_{\mu=1}^{m} \omega_{\mu}<\rho .
\end{gathered}
$$


(b) There are constants $\alpha_{1}, \ldots, \alpha_{m}$ with $\sum_{\mu=1}^{m} \alpha_{\mu} \geqq m$ so that $(1 / m) \sum_{\mu=1}^{m} \omega_{\mu}=\rho$, (57) holds and

$$
\begin{array}{cc}
\Omega_{\mu}<\infty \quad & (\mu=1, \ldots, m), \\
\Delta_{\mu}=0 \quad(\mu=1, \ldots, m), \\
\prod_{\mu=1}^{m} \Omega_{\mu}<\frac{\sigma^{m}}{2(2 m(1+a))^{m}} .
\end{array}
$$

For example, (57) will hold with $a=1$ if $h_{v}$ is nondecreasing so that $D_{v} /\left(v_{0}+v\right)$ is nondecreasing; $\alpha_{\mu} / m+\rho-\omega_{\mu} \geqq 0$ and $\alpha_{\mu} \geqq 1$ for $\mu=1, \ldots, m$.

Proof. Let us prove part (b). Let $\varepsilon>0$ and let $\Omega_{\mu}^{\prime}=\Omega_{\mu}+\varepsilon$. Then $0<\Omega_{\mu}^{\prime}<\infty$ and

$$
\log 3 H\left(f_{\mu}\left(z_{v}\right)\right) \leqq \frac{\Omega_{\mu}^{\prime} \nu^{\omega_{\mu}} \log \nu}{D_{\nu^{\prime}}^{\alpha^{\prime m}}\left(d_{v+1}^{*}\right)^{\alpha_{\mu}}}=p(\nu)
$$

for sufficiently large $\nu$. Assume that (62) holds for $\nu>\nu_{0}$. By (60) we have

$$
\lim _{\nu \rightarrow \infty} p(\nu)=\infty \text {. }
$$

Therefore we can choose $n$ sufficiently large and so that $\nu \leqq \nu_{0}+n$ implies that $p(v) \leqq p\left(\nu_{0}+n\right)$. For such an $n$ we have by (62)

for

$$
\log 3 H\left(f_{\mu}\left(z_{v}\right)\right) \leqq p\left(v_{0}+n\right)
$$

Let

$$
v=v_{0}+1, \ldots, v_{0}+n
$$

$$
t_{\mu}=\left[\left(2 \prod_{\mu=1}^{m} \Omega_{\mu}^{\prime}\right)^{1 / m} / \Omega_{\mu}^{\prime} \cdot D_{n^{\mu} / n}^{\alpha}\left(\nu_{0}+n\right)^{\rho-\omega_{\mu}}\left(d_{n+1}^{*}\right)^{\alpha_{\mu}-1}\right]
$$

and

$$
\tau(n)=\left(n^{\rho} \log n\right) / d_{n+1}^{*}
$$

Then by (57), $t_{\mu}$ is a nondecreasing function of $n$. From (59) we obtain $d_{n+1}^{*}=o\left(n^{o}\right)$ and consequently $\tau(n)$ is nondecreasing.

Once again we obtain integers $c_{\tau_{1} \cdots \tau_{m}}$ not all zero for which

$$
\left|c_{\tau_{1} \cdots \tau_{m}}\right| \leqq \exp \left[m\left(2 \prod_{\mu=1}^{m} \Omega_{\mu}^{\prime}\right)^{1 / m} \tau(n)+o(\tau(n))\right]
$$

and this upper bound is a nondecreasing function of $n$. If we assume that $\phi(z)=0$ for $z=z_{v_{0}+1}, \ldots, z_{v_{0}+n_{1}}$ where $n_{1} \geqq n$, we conclude that

$$
\begin{gathered}
\left|\phi\left(z_{v_{0}+n_{1}+1}\right)\right| \leqq \exp \left[m\left(2 \prod_{\mu=1}^{m} \Omega_{\mu}^{\prime}\right)^{1 / m} \tau\left(n_{1}\right)-(\sigma-\varepsilon) n_{1}^{\rho} \log n_{1}+o\left(n_{1}^{\rho} \log n_{1}\right)\right], \\
K \leqq \exp \left[a m\left(2 \prod_{\mu=1}^{m} \Omega_{\mu}^{\prime}\right)^{1 / m} \tau\left(n_{1}\right)+o\left(\tau\left(n_{1}\right)\right)\right]
\end{gathered}
$$


and

Consequently,

$$
\left|\phi^{(\delta)}\left(z_{v_{0}+n_{1}+1}\right)\right| \leqq \exp \left[(1+a) m\left(2 \prod_{\mu=1}^{m} \Omega_{\mu}^{\prime}\right)^{1 / m} \tau\left(n_{1}\right)+o\left(\tau\left(n_{1}\right)\right)\right] .
$$

$$
\begin{aligned}
& \left|N\left(K \phi\left(z_{v_{0}+n_{1}+1}\right)\right)\right| \\
& \quad \leqq \exp \left[\left\{2 m(1+a)\left(2 \prod_{\mu=1}^{m} \Omega_{\mu}^{\prime}\right)^{1 / m}-(\sigma-\varepsilon)\right\} n_{1}^{\rho} \log n_{1}+o\left(n_{1}^{\rho} \log n_{1}\right)\right]<1
\end{aligned}
$$

if $n>n_{0}$ and $\varepsilon$ is sufficiently small. This completes the proof of (b). Part (a) is established in a similar manner.

Conditions (a) and (b) become significant in case the inequality in (36) is replaced by equality. Condition (b) covers Theorem $\mathrm{I}\left({ }^{3}\right)$ and Theorem III.

A number of interesting corollaries can be obtained. Let $m=2, f_{1}(z)=g(z)$, $f_{2}(z)=z, d_{v}=d, \alpha_{1}=2 \rho$ and $\alpha_{2}=0$.

COROllary I. Let $g(z)$ be analytic at $z=0$. Let $\left\{z_{v}\right\}$ be a sequence of distinct algebraic numbers of bounded degree with $\lim _{v \rightarrow \infty} z_{v}=0$. Suppose that $g\left(z_{v}\right)$ is algebraic of bounded degree. Let $\operatorname{deg} Q\left(g\left(z_{v}\right), z_{v}\right) \leqq d$. Then either of the following conditions (c) and (d) implies that $g(z)$ is algebraic (in the arithmetic sense).

$$
\limsup _{v \rightarrow \infty} \frac{\log \log 3 H\left(z_{v}\right)}{\log v}+\limsup _{v \rightarrow \infty} \frac{\log \log 3 H\left(g\left(z_{v}\right)\right)}{\log v}<2 \rho-1 \text {. }
$$

$$
\begin{gathered}
A=\limsup _{v \rightarrow \infty} \frac{\log H\left(g\left(z_{v}\right)\right)}{\nu^{\rho} \log v}<\infty . \\
B=\limsup _{v \rightarrow \infty} \frac{\log H\left(z_{v}\right)}{\log v}<\infty . \\
A B<\frac{\sigma^{2}}{128 d^{3 \rho}} .
\end{gathered}
$$

In particular, if the requirement (63) is strengthened, we obtain the following simpler result.

COROllary I'. St:ppose $\sigma \neq 0$. Then the conclusion of Corollary I holds under the same hypotheses but with (63), (64), and (65) replaced by

and

$$
\lim _{v \rightarrow \infty} \frac{\log H\left(g\left(z_{v}\right)\right)}{\nu^{\rho} \log v}=0
$$

$$
\limsup _{v \rightarrow \infty} \frac{\log H\left(z_{v}\right)}{\log v}<\infty .
$$

We now turn to the case of unbounded degree.

$\left({ }^{3}\right)$ Except for the fact that the resulting upper bound for $y$ in (8) is smaller due to the cruder type of Diophantine equation argument used in the proof of Theorem VII. 
COROllaRY II. Let $g(z)$ be analytic at $z=0$. Let $\left\{z_{v}\right\}$ be a sequence of distinct algebraic numbers with $\lim _{v \rightarrow \infty} z_{v}=0$. Suppose that $g\left(z_{v}\right)$ is algebraic for $v>v_{0}$. Let $\operatorname{deg} Q\left(g\left(z_{v_{0}+v}\right), z_{v_{0}+v}\right) \leqq d_{v}, \operatorname{deg} z_{v} \operatorname{deg} g\left(z_{v}\right) \leqq h_{v}$ and

$$
D_{v}=\sum_{x=v_{0}+v}^{\nu_{0}+n} h_{x} .
$$

Suppose that $\sigma \neq 0, d_{v}$, and $h_{v}$ are nondecreasing,

$$
\lim _{v \rightarrow \infty} \frac{D_{\nu}^{\rho}\left(d_{v+1}\right)^{2 \rho} \log 3 H\left(g\left(z_{v}\right)\right)}{v^{2 \rho} \log \nu}=0
$$

and

$$
\limsup _{v \rightarrow \infty} \frac{\log H\left(z_{v}\right)}{\log \nu}<\infty .
$$

Then $g(z)$ is algebraic (in the arithmetic sense).

Corollary I covers Theorem I. Corollaries I and I' generalize Içen's Theorem I in the case where $\sigma \neq 0, H\left(z_{v}\right)$ grows at most like a power of $\nu$ and $g(z)$ is a function of a complex variable. Corollary II covers Theorem III and Corollary I'.

\section{BIBLIOGRAPHY}

O. S. IÇEN

1. Eine Verallgemeinerung und Ubertragung der Schneiderschen Algebraizitätskriterien ins p-adische mit Anwendung auf einen Transzendenzbeweis im p-adischen, J. Reine Angew. Math. 198 (1957), 28-55.

2. Eine weitere Verallgemeinerung eines Schneiderschen Algebraizitätskriteriums, Rev. Fac. Sci. Univ. Istanbul Sér. A 21 (1956), 155-187; Fehlerverzeichnis zur Arbeit, ibid. (1957), 261.

S. LANG

1. Diophantine geometry, Interscience Tracts in Pure and Applied Mathematics No. 11, Interscience, New York, 1962.

W. J. LE Veque

1. Topics in number theory, Vol. II, Addison-Wesley, Reading, Massachusetts, 1956.

TH. SCHNEIDER

1. Zur Charakterisierung der algebraischen und der rationalen Funktionen durch ihre Funktionswerte, Acta Math. 86 (1951), 57-70.

2. Zur Charakterisierung algebraischer Funktionen mit Hilfe des Eisensteinschen States, Math. Z. 60 (1954), 98-108.

3. Einführung in die transzendenten Zahlen, Springer-Verlag, Berlin, 1957.

UNIVERSITy OF CALIFORNIA, Irvine, CaLIFornia 